\title{
Arsenic, DNA damage, and cancers of bladder and kidney - a long-term follow-up of residents in arseniasis endemic area of north-eastern Taiwan
}

\author{
S.-L. Wang ${ }^{1}$, S.-F. Tsai ${ }^{1}$, L.-I. Hsu ${ }^{2}$, C.-J. Chen ${ }^{2}$, K.-H. Hsu ${ }^{3} \&$ H.-Y. Chiou ${ }^{4}$ \\ ${ }^{1}$ National Institute of Environmental Heath Sciences, National Health Research Institutes, Miaoli, Taiwan \\ ${ }^{2}$ Genomics Research Centre, Academia Sinica, Taipei, Taiwan \\ ${ }^{3}$ Department of Health Care Management, Chang-Gung University, Taoyuan, Taiwan \\ ${ }^{4}$ Department of Public Health, Taipei Medical University, Taipei, Taiwan
}

\begin{abstract}
The aim is to assess dose-response association between arsenic exposure, DNA damage biomarkers, and the incidence of bladder and kidney cancers. A total of 8102 men and women from 3901 households have been enrolled in 1991-1994, and followed in 2011-2014. The data collected included well water consumption, habits of cigarette smoking, alcohol consumption, exercise and diet, through standardized personal interview. The individual urinary arsenic species were quantified using high-performance liquid chromatography-inductively coupled plasma/mass spectrometry (HPLC-ICP/MS). For assessment of oxidative and methylated DNA lesions and depletion, urinary 8-oxo-7,8-dihydro-2'-deoxyguanosine (8-oxodG) and N7-methylguanine (N7-MeG) were measured respectively, using liquid chromatography/tandem mass spectrometry (LC-MS/MS). The National Cancer Registry Data using the pathology finding defined bladder and kidney cancers. Urinary levels of the two DNA adduct increased significantly with increasing urinary arsenic level (iAs+MMA+DMA) in both men ( $\beta=0.82, \beta=0.34$ for 8-oxodG and N7-MeG, respectively, $\mathrm{p}<0.0001$ for both) and women $(\beta=1.03,0.38$, $\mathrm{p}<0.0001)$ adjusted for potential confounders. Incidence rate of bladder and kidney cancer using person-years tended to be the highest for higher urinary inorganic and methylated arsenic with higher DNA adduct level than the medians. It is suggested that subjects with high arsenic exposure experienced further cancer risk with high level of DNA damage biomarker.
\end{abstract}

\section{INTRODUCTION}

Arsenic is ubiquitous and becoming one of the largest environmental health concerns in the area where groundwater is needed as source of drinking water, or arsenic is prevalent in air pollutants. High inorganic arsenic exposure has been found to be related to various cancers and cardiovascular diseases (Lee \& Yu, 2016; Rorbach-Dolata et al., 2015; Yager et al., 2013). The aim of this study is to assess dose-response association between arsenic exposure, DNA damage biomarkers, and the incidence of bladder and kidney cancers.

\section{METHODS}

\subsection{Subjects}

A total of 8102 men and women from 3901 households have been enrolled in 1991-1994 and followed in 2011-2014. The data collected included well water consumption, habits of cigarette smoking, alcohol consumption, exercise and diet, and personal and family history of major diseases through standardized personal interview.

\subsection{Measurements}

The individual urinary arsenic species were quantified using high-performance liquid chromatographyinductively coupled plasma/mass spectrometry (HPLC-ICP/MS).

For assessment of oxidative and methylated DNA lesions and depletion, urinary 8-oxo-7,8-dihydro-2'deoxyguanosine (8-oxodG) and N7-methylguanine (N7-MeG) were measured respectively, using liquid chromatography/tandem mass spectrometry (LC-MS/MS). The National Cancer Registry Data using the pathology finding defined bladder and kidney cancers.

\section{RESULTS AND DISCUSSION}

Urinary levels of the two DNA adduct increased significantly with increasing urinary arsenic level (iAs+MMA+DMA) in both men $(\beta=0.82, \beta=0.34$ for 8-oxodG and N7-MeG, respectively, $\mathrm{p}<0.0001$ for both) and women $(\beta=1.03,0.38, \mathrm{p}<0.0001)$ adjusted for potential confounders of age and cigarette smoking. Incidence rate of bladder and kidney cancer using person-years tended to be the highest for higher 
urinary inorganic and methylated arsenic with higher DNA adduct level than the medians.

\section{CONCLUSIONS}

It is suggested that subjects with high arsenic exposure experienced further cancer risk with high level of DNA damage biomarker.

\section{ACKNOWLEDGEMENTS}

Grant from Ministry of Science and Technology (MOST) Taiwan.

\section{REFERENCES}

Lee, C.H. \& Yu, H.S. 2016. Role of mitochondria, ROS, and DNA damage in arsenic induced carcinogenesis. Front Bioscan (Schol Ed)8: 312-320.

Rorbach-Dolata, A., Marchewka, Z. \& Piwowar, A. 2015. The biochemical carcinogenesis of selected heavy metals in bladder cancer. Postepy Biochem. 61(2): 176-182.

Yager, J.W., Gentry, P.R., Thomas, R.S., Pluta, L., Efremenko, A., Black, M., Arnold, L.L., McKim, J.M., Wilga, P. \& Gill, G. 2013. Evaluation of gene expression changes in human primary uroepithelial cells following 24-hr exposures to inorganic arsenic and its methylated metabolites. Environ. Mol. Mutagen. 54(2): 82-98. 